\title{
Integrated DNA/RNA targeted genomic profiling of diffuse large B-cell lymphoma using a clinical assay
}

Andrew M. Intlekofer (10,2,3,4, Erel Joffe (1) 1,2, Connie L. Batlevi ${ }^{1,2}$, Patrick Hilden (10), Jie He ${ }^{6}$, Venkatraman E. Seshan ${ }^{5}$, Andrew D. Zelenetz ${ }^{1,2}$, M. Lia Palomba ${ }^{1,2}$, Craig H. Moskowitz ${ }^{1,2}$, Carol Portlock,2, David J. Straus ${ }^{1,2}$, Ariela Noy ${ }^{1,2}$, Steven M. Horwitz ${ }^{1,2}$, John F. Gerecitano ${ }^{1,2}$, Alison Moskowitz ${ }^{1,2}$, Paul Hamlin 1,2, Matthew J Matasar ${ }^{1,2}$, Anita Kumar ${ }^{1,2}$, Marcel R. van den Brink7, Kristina M. Knapp³, Janine D. Pichardo ${ }^{8}$, Michelle K. Nahas ${ }^{6}$, Sally E. Trabucco (1) ${ }^{6}$, Tariq Mughal ${ }^{6}$, Amanda R. Copeland ${ }^{1,2}$, Elli Papaemmanuil ${ }^{4}$, Mathai Moarii ${ }^{4}$, Ross L. Levine ${ }^{2,3,4,9}$, Ahmet Dogan ${ }^{8}$, Vincent A. Miller ${ }^{6}$ and Anas Younes ${ }^{1,2}$

\begin{abstract}
We sought to define the genomic landscape of diffuse large B-cell lymphoma (DLBCL) by using formalin-fixed paraffinembedded (FFPE) biopsy specimens. We used targeted sequencing of genes altered in hematologic malignancies, including DNA coding sequence for 405 genes, noncoding sequence for 31 genes, and RNA coding sequence for 265 genes (FoundationOne-Heme). Short variants, rearrangements, and copy number alterations were determined. We studied 198 samples (114 de novo, 58 previously treated, and 26 large-cell transformation from follicular lymphoma). Median number of GAs per case was 6 , with $97 \%$ of patients harboring at least one alteration. Recurrent GAs were detected in genes with established roles in DLBCL pathogenesis (e.g. MYD88, CREBBP, CD79B, EZH2), as well as notable differences compared to prior studies such as inactivating mutations in TET2 (5\%). Less common GAs identified potential targets for approved or investigational therapies, including BRAF, CD274 (PD-L1), IDH2, and JAK1/2. TP53 mutations were more frequently observed in relapsed/refractory $D L B C L$, and predicted for lack of response to first-line chemotherapy, identifying a subset of patients that could be prioritized for novel therapies. Overall, $90 \%(n=169)$ of the patients harbored a GA which could be explored for therapeutic intervention, with 54\% $(n=107)$ harboring more than one putative target.
\end{abstract}

\section{Key points}

- This study demonstrates the applicability of CLIAcompliant targeted FFPE sequencing for large-scale clinical trials.
- TP53mut is the main predictor of refractoriness or early relapse.

\section{Introduction}

The ability to identify and therapeutically target patientspecific genomic alterations has made precision oncology a reality for several types of cancer ${ }^{1}$. Unfortunately, in

\footnotetext{
Correspondence: Anas Younes (younesa@mskcc.org)

${ }^{1}$ Lymphoma Service, Department of Medicine, Memorial Sloan Kettering Cancer Center, New York, NY, USA

${ }^{2}$ Department of Medicine, Weill Cornell Medical College, New York, NY, USA Full list of author information is available at the end of the article

This work was presented in part at the 56th American Society of Hematology (ASH) Annual Meeting, San Francisco, CA, 6-9 December 2014.

These authors contributed equally: Andrew M. Intlekofer, Erel Joffe.
} 
aggressive lymphomas, no approved genomic biomarkerdriven therapies are standard of care. The problem is exemplified by diffuse large B-cell lymphoma (DLBCL) where, despite a relapse rate of over $30 \%, \mathrm{RCHOP}$ is being administered as an almost uniform first-line of care, over two decades since it was introduced ${ }^{2}$. Thus, there is an unmet need to develop genomic biomarker-driven therapeutics, to improve outcomes for patients with DLBCL.

Next-generation sequencing studies have produced a vast array of data regarding the underlying genomic alterations (GAs) that characterize DLBCL. These demonstrate a striking genetic heterogeneity that likely accounts for the observed variability in clinical phenotype $^{3-7}$. Recurrent alterations have been identified in over 300 genes, none of which pathognomonic, as all occur at frequencies $<30 \%$, usually $<10 \%{ }^{7}$. Identifying the clinical implications of these alterations requires large cohorts, and the integration of several testing modalities (e.g. DNA sequencing to identify short nucleotide variations (SNVs) and copy number alterations (CNAs), and RNA sequencing for gene rearrangements) ${ }^{8}$. In this regard, most genomic studies in DLBCL have been carried out in the research setting, often implementing assays such as whole genome or whole exome sequencing using fresh frozen biopsy specimens ${ }^{7}$. These may be prohibitively resource intensive for adaptation in large-scale clinical trials or in everyday practice. In these settings, integrated hybridization capture of both DNA and RNA using formalin-fixed paraffin-embedded (FFPE) specimens may be most appropriate $^{7,9}$. Potential advantages of this approach include: (1) simultaneous detection of all classes of GAs including short variants (base substitutions and small indels), CNAs, and gene rearrangements/fusions; (2) sensitive detection of fusion transcripts involving the genes of interest due to inclusion of the RNA sequencing component; (3) flexibility in sample acquisition, storage and transportation when using FFPE; (4) high depth of sequencing coverage to enhance detection of rare variants even in samples with extensive non-malignant stromal and immune cell contamination; (5) streamlined bioinformatics; and (6) compliance with The Clinical Laboratory Improvement Amendments (CLIA) standard. However, it remains to be established what spectrum of GAs will be observed in clinical FFPE DLBCL specimens, how specific GAs will correlate with clinical and pathologic phenotype and with patient outcomes, and how this information can be incorporated in clinical care.

Herein, we describe the application of commercially available, CLIA-compliant, integrated DNA and RNA targeted sequencing panel (FoundationOne-Heme) to a retrospective cohort of 198 FFPE DLBCL specimens for the identification of GAs with potential clinical significance.

\section{Materials and methods Study population}

Archived FFPE biopsy specimens from 198 patients with DLBCL were obtained with approval from the Memorial Sloan Kettering Cancer Center (MSKCC) Institutional Review Board. Biopsies were collected between 1989 and 2012. Inclusion criteria were histologically confirmed DLBCL with appropriate patient consent to perform genomic sequencing. Germinal center B-cell-like (GCB) or non-GCB cell-of-origin (COO) was assessed by immunohistochemistry (IHC) according to the Hans algorithm ${ }^{8}$. Baseline demographics and survival data were extracted from the clinical record.

\section{Sample preparation and sequencing}

Samples were sequenced using the FoundationOneHeme platform that uses DNA sequencing to interrogate the entire coding sequence of 406 genes, selected introns of 31 genes involved in rearrangements, and utilizes RNA sequencing to interrogate 265 genes known to be somatically altered in human hematologic malignancies ${ }^{10}$. Detailed protocols for DNA and RNA extraction, cDNA synthesis, library construction, and hybrid selection as well as a survey of methodological validation tests, have been recently published, and are detailed in the supplementary methods and supplementary table $1^{10,11}$. In brief, specimens were reviewed by a pathologist to confirm $\geq 20 \%$ tumor nuclei and a tissue volume of $\geq 2 \mathrm{~mm}^{3}$. We used $20 \%$ tumor content as the minimal requirement, having demonstrated in a previous validation study that the pipeline approaches a sensitivity of $100 \%$ for SNVs and CNAs above this cutoff ${ }^{10}$. Genomic DNA and RNA were extracted and fragmented to $\sim 200 \mathrm{bp}$ fragment size. Samples were tested to ensure sufficient DNA yield $(50-200 \mathrm{ng})$ and RNA yield $(\geq 3.5 \mathrm{ng} / \mu \mathrm{L})$. Sequencing was performed with the Illumina HiSeq2500 system using $49 \times 49$ paired-end reads. Resultant sequences were analyzed for single nucleotide variants (SNVs-base substitutions and small indels), CNAs and rearrangements. Samples with median coverage $<150 \times$ were considered failed and excluded from analysis. Known germline variants (per 1000 Genomes Project) were removed ${ }^{10,11}$. Significant non-synonymous variants were defined as any somatic alteration annotated in the COSMIC database (v62), as well as clear inactivating mutations (i.e. truncations or deletions) in established tumor suppressor genes $^{10,11}$. The mutant allele frequency cutoff used for known somatic variants was $1 \%$; $5 \%$ for potential driver somatic variants; $3 \%$ for previously described indels; and $10 \%$ for potential driver indels. Gene amplifications/gains were defined at a copy number $\geq 6$, and gene losses as copy number of $0^{10,11}$. For rearrangement identification we required a minimum of ten chimera reads for known fusions and 50 for potential driver rearrangements. Any 
aberration not meeting the aforementioned criteria was defined as Unknown Significance (UKS). Sequencing data are publicly available in an interactive format through the cBioPortal (www.cbioportal.org).

Individual genes were grouped together by the biologic pathways in which they operate (supplementary table $2)^{12,13}$. Actionable variants and pathways were defined by the presence of GAs predictive of response to an FDAapproved drug or an experimental agent in clinical trial ${ }^{14}$. These data were derived by a review of literature and publicly accessible databases including OncoKB, the FDA Pharmacogenomic Biomarkers in Drug Labeling, GeneCards, and clinicaltrials.gov ${ }^{12-17}$. Variant actionability was graded based on the OncoKB criteria. Level 1 was defined as alterations recognized by the FDA as predictive of response to an approved drug in DLBCL. Level 2 included non-FDA predictive biomarkers for response in DLBCL (2A) or FDA-approved biomarkers for response in a different malignancy (2B). Level 3 includes alterations supported by compelling data from clinical trials in DLBCL (3A) or another malignancy (3B). Level 4 are candidate biomarkers for response based on early clinical or preclinical studies (supplementary excel file 2$)^{14}$.

\section{Statistical analysis}

Descriptive statistics are provided for all genes dichotomously (i.e. presence/absence of any alteration). Differences in alteration frequency between groups were determined using Fischer's exact test, with differences in the total number of alterations across groups assessed using a Wilcoxon rank-sum test. Clustering analysis was done based on the Jaccard distance using the Ward D method. Analyses for response to treatment and survival were performed in the subset of patients with de novo disease treated with RCHOP (rituximab, cyclophosphamide, adriamycin, vincristine, steroids) or RCHOPlike chemotherapy. For the purpose of these analyses, tFL not previously treated was included with the de novo group, reasoning that in clinical practice the distinction between tFL at first diagnosis and DLBCL is not made easily, such that both conditions are treated similarly as de novo DLBCL and have comparable outcomes ${ }^{18}$. Median follow-up was estimated using the reverse Kaplan-Meier method. Overall and progression-free survival (OS/PFS) were defined as the time from initiation of frontline treatment until death of any cause or disease progression or death (for PFS), censoring at the end of follow-up. Differences in OS and PFS between groups were assessed using the Kaplan-Meier method as well as univariate Cox proportional hazards regression models. Where applicable we adjusted for false discovery (FDR) using the Benjamini-Hochberg approach. Analyses were done in $\mathrm{R}$ 3.4.0 ( $\mathrm{R}$ foundation, Austria).

\section{Results}

Of 219 FFPE DLBCL samples attempted, 214 were successfully sequenced, indicating a success rate of $98 \%$. Sixteen cases were excluded from the analysis (for inadequate clinical data, primary central nervous system lymphoma or large-cell transformation from indolent lymphomas other than FL), leaving 198 cases for this analysis: 114 cases were from newly diagnosed untreated patients (de novo), 58 from previously treated patients, and 26 from tFL cases. Cell of origin was determined in 177 cases, with $48 \%(n=95)$ classified as GCB and $41 \%(n$ $=82$ ) as non-GCB. Of the 114 patients sequenced at diagnosis, $30 \%(n=35)$ were refractory to first-line treatment or subsequently relapsed during follow-up. The median unique sequencing coverage was $555 \times$ [476-656] for DNA and median total pairs for RNA were $>20 \times 10^{6}$.

\section{Genomic alterations and pathways landscape}

The median number of GAs per case was 6 , with $97 \%$ of patients harboring at least one alteration (Table 1, supplementary figure 1 and supplementary excel file 1 ). The most commonly identified SNVs were in KMT2D (MLL2; $31 \%, n=62)$, TP53 (24\%, $n=48)$, MYD88 (18\%, $n=36)$, CREBBP $(18 \%, n=35)$, and B2M (Beta-2-microglobulin; $17 \% ; n=33$ ) (Fig. 1, Table 1). CNAs were identified in $42 \%(n=84)$ of cases, involving 37 different genes. The most frequently identified losses were $C D K N 2 A$ and/or $C D K N 2 B$ (20\% combined, $n=40)$, while the most frequent gene amplifications were observed in $R E L(8 \%, n=$ 16), CD274 ( $3 \% ; n=6)$, and MCL1 $(3 \%, n=6)$. Rearrangements (trans) were detected in $57 \%(n=112)$ of cases involving 61 different genes. As expected, most involved the translocation of $B C L 2, B C L 6$, or $M Y C$ to the immunoglobulin heavy chain $(I G H)$ enhancer (supplementary table 3). Deletion of $C D K N 2 B$ was always accompanied by deletion of CDKN2A (though not vice versa) and associated with CD79Bmut, MYD88mut, PIM1mut, and PRDM1mut (Fig. 2, supplementary figure 2). CDKN2Bdel and CDKN2Adel were mutually exclusive with TP53mut $(p<0.001)$ as were BCL10mut $(p=0.04)$ and CD58mut $(p=0.04)$. A cluster of BCL2trans and KMT2Dmut corresponded with a GCB subtype and with high rates of TP53mut, EZH2mut, and TNFRSF14mut ( $p=0.002$; Fig. 2 ). Of note, the largest cluster of 80 patients (40\%) did not have a distinct genomic signature.

Patients with R/R disease and tFL had higher rates of TP53mut compared to de novo patients $(16 \% n=18$ de novo, $33 \% n=19 \mathrm{R} / \mathrm{R}$; and $42 \% n=11 \mathrm{tFL} ; p=0.02 \mathrm{R} / \mathrm{R}$ vs. de novo), and of KMT2Dmut (19\% $n=22$ de novo, $38 \% n=22 \mathrm{R} / \mathrm{R}$; and $69 \% n=18 \mathrm{tFL} ; p=0.01 \mathrm{R} / \mathrm{R}$ vs. de novo) (Table 1). Further, $R / R$ and tFL cases were enriched for translocations $(45 \% n=51$ de novo, $71 \% n=41 \mathrm{R} / \mathrm{R}$; and $77 \% n=20$ tFL; $p=0.002$ for $\mathrm{R} / \mathrm{R}$ vs. de novo). These 
Table 1 Summary of key genomic alterations by disease status at time of sequencing

\begin{tabular}{|c|c|c|c|c|c|c|}
\hline & {$[A L L]$} & de novo & $\mathrm{R} / \mathrm{R}$ & $\mathrm{tFL}$ & $p$ value* & $\mathrm{BH} p^{*}$ \\
\hline & 198 & 114 & 58 & 26 & & \\
\hline \multicolumn{7}{|l|}{ Cell of origin } \\
\hline GCB & 95 (48.0\%) & $51(44.7 \%)$ & $26(44.8 \%)$ & $18(69.2 \%)$ & 0.602 & \\
\hline Non-GCB & $82(41.4 \%)$ & $51(44.7 \%)$ & $23(39.7 \%)$ & $8(30.8 \%)$ & & \\
\hline NA & $21(10.6 \%)$ & $12(10.5 \%)$ & $9(15.5 \%)$ & $0(0.00 \%)$ & & \\
\hline SNVS & $191(96.5)$ & $108(94.7)$ & $57(98.3)$ & $26(100.0)$ & 0.426 & \\
\hline SNVs per/pt. (min, max) & $4(0,9)$ & $4(0,9)$ & $4(0,9)$ & $4.5(1,9)$ & 0.640 & \\
\hline SNVs of UKS per/pt. & $15.5(3,45)$ & $16(3,45)$ & $15.5(4,28)$ & $13.5(6,23)$ & 0.894 & \\
\hline Amplifications & $36(18.2)$ & $20(17.5)$ & $10(17.2)$ & $6(23.1)$ & 1.000 & \\
\hline Deletions & $57(28.8)$ & $28(24.6)$ & $20(34.5)$ & 9 (34.6) & 0.233 & \\
\hline Translocations & $112(56.6)$ & $51(44.7)$ & $41(70.7)$ & $20(76.9)$ & 0.002 & \\
\hline Total number of GAs & $6(0,13)$ & $5(0,13)$ & $6(0,13)$ & $7(1,11)$ & 0.078 & \\
\hline KMT2D & $62(31.3 \%)$ & 22 (19.3\%) & $22(37.9 \%)$ & $18(69.2 \%)$ & 0.014 & 0.253 \\
\hline CDKN2A & $54(27.3 \%)$ & $28(24.6 \%)$ & $20(34.5 \%)$ & $6(23.1 \%)$ & 0.233 & 0.726 \\
\hline TP53 & $48(24.2 \%)$ & $18(15.8 \%)$ & 19 (32.8\%) & $11(42.3 \%)$ & 0.018 & 0.253 \\
\hline BCL2 & 46 (23.2\%) & $16(14.0 \%)$ & 15 (25.9\%) & 15 (57.7\%) & 0.090 & 0.501 \\
\hline BCL6 & 37 (18.7\%) & $21(18.4 \%)$ & 13 (22.4\%) & $3(11.5 \%)$ & 0.675 & 0.934 \\
\hline MYD88 & 36 (18.2\%) & $21(18.4 \%)$ & 13 (22.4\%) & 2 (7.69\%) & 0.675 & 0.934 \\
\hline CREBBP & 35 (17.7\%) & 16 (14.0\%) & 9 (15.5\%) & $10(38.5 \%)$ & 0.975 & 1.000 \\
\hline B2M & 33 (16.7\%) & 19 (16.7\%) & $8(13.8 \%)$ & $6(23.1 \%)$ & 0.789 & 0.934 \\
\hline CDKN2B & $32(16.2 \%)$ & 17 (14.9\%) & $10(17.2 \%)$ & 5 (19.2\%) & 0.861 & 0.964 \\
\hline TNFAIP3 & $24(12.1 \%)$ & 15 (13.2\%) & 7 (12.1\%) & 2 (7.69\%) & 1.000 & 1.000 \\
\hline $\mathrm{EZH} 2$ & 21 (10.6\%) & $13(11.4 \%)$ & 4 (6.90\%) & $4(15.4 \%)$ & 0.505 & 0.934 \\
\hline PIM1 & 20 (10.1\%) & 11 (9.65\%) & 8 (13.8\%) & 1 (3.85\%) & 0.574 & 0.934 \\
\hline TNFRSF14 & 20 (10.1\%) & $9(7.89 \%)$ & 4 (6.90\%) & 7 (26.9\%) & 1.000 & 1.000 \\
\hline CARD11 & 19 (9.60\%) & 7 (6.14\%) & $5(8.62 \%)$ & 7 (26.9\%) & 0.541 & 0.934 \\
\hline ARID1A & 16 (8.08\%) & 9 (7.89\%) & 7 (12.1\%) & 0 (0.00\%) & 0.540 & 0.934 \\
\hline REL & 16 (8.08\%) & 10 (8.77\%) & 3 (5.17\%) & $3(11.5 \%)$ & 0.547 & 0.934 \\
\hline CD79B & 15 (7.58\%) & $9(7.89 \%)$ & $6(10.3 \%)$ & 0 (0.00\%) & 0.801 & 0.934 \\
\hline FAS & 15 (7.58\%) & 9 (7.89\%) & $6(10.3 \%)$ & 0 (0.00\%) & 0.801 & 0.934 \\
\hline
\end{tabular}

were mainly $I G H: B C L 2$ rearrangements for $\mathrm{tFL}$ (75\% of cases), while among $\mathrm{R} / \mathrm{R}$ patients $37 \%(n=15)$ had a BCL2trans, $27 \%(n=11)$ had a BCL6trans, and $12 \%(n=$ 5) had a MYCtrans.

These observations corresponded to a trend towards higher overall rates of abnormalities in tumor suppressors pathways that includes TP53mut and CDKN2Bdel (54\% $n$ $=61$ de novo, $76 \% n=44 \mathrm{R} / \mathrm{R}$, and $69 \% n=18 \mathrm{tFL}, p=$ 0.007 ) and in the epigenetic histone modification pathway which includes KMT2Dmut (47\% $n=41$ non-relapsing,
$64 \% n=37 \mathrm{R} / \mathrm{R}$, and $89 \% n=23 \mathrm{tFL}, p=0.008$ ) (Table 2). Of note, after correction for false discovery, none of these differences remained statistically significant. As expected, BCL2trans were more common in GCB compared to nonGCB $(40 \% n=38$ vs. $5 \% n=4, p<0.001)$ as were CREBBPmut (27\% $n=26$ vs. $6 \% \quad n=5, p<0.001)$, KMT2Dmut ( $43 \% n=41$ vs. $21 \% n=17, p=0.003)$, and TNFRSF14 $(17 \% \quad n=16 \quad$ vs. $4 \% \quad n=3, \quad p=0.01)$. CD79Bmut were observed solely in non-GCB ( 0 vs. $16 \% n$ $=13, p<0.001)$, as was an enrichment for BCL6trans 


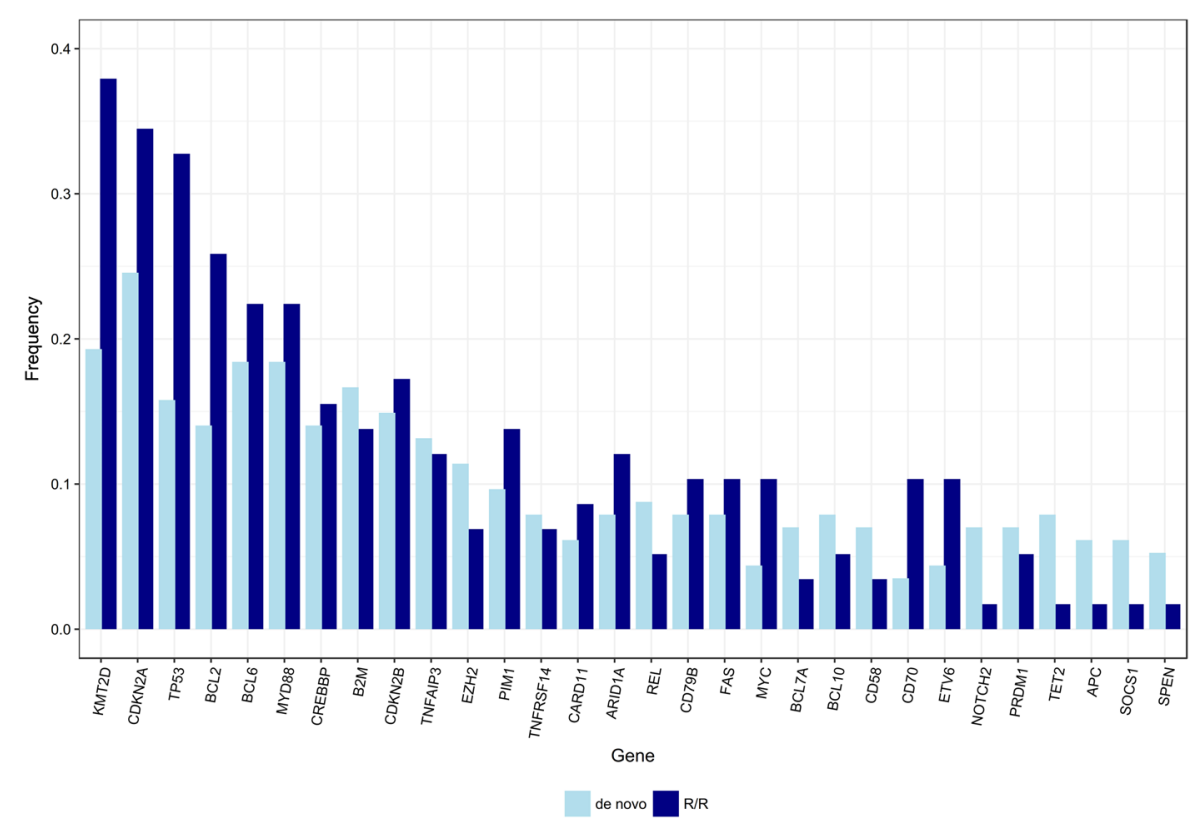

Fig. 1 Genomic alterations in de novo vs. R/R disease. Bar plot of genomic alterations present in $\geq 5 \%$ of the subjects by order of frequency and by R/R status. tFL cases are not presented. The significantly different GAs were TP53mut $(p=0.02)$ and KMT2Dmut $(p=0.01)$

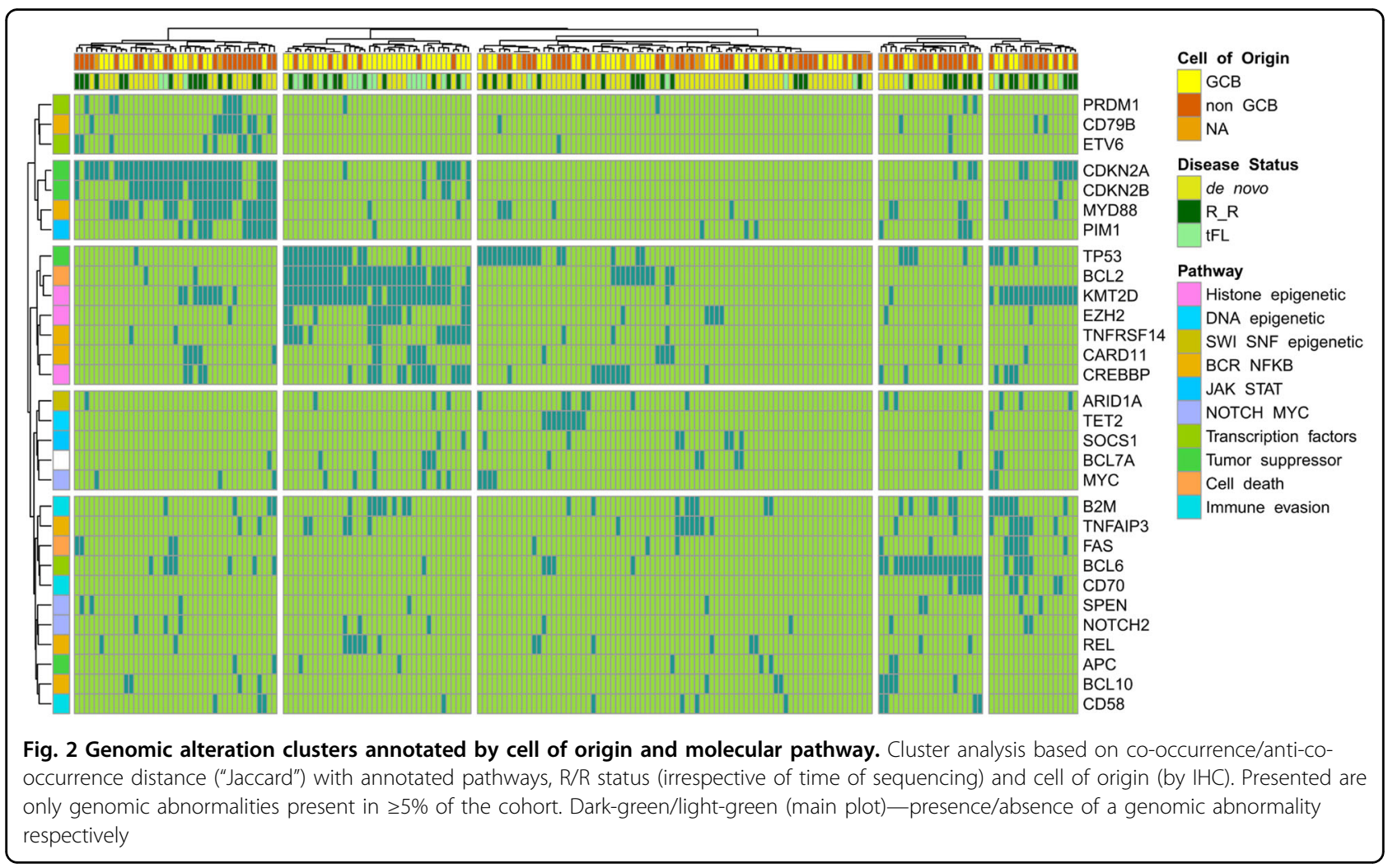

$(11 \% n=10$ vs. $27 \% n=22, p=0.01$ ) (supplementary table 4 and Fig. 3). We further observed an enrichment in MYD88mut, ETV6mut, and PRDM1mut among non-GCB and EZH2mut among GCB tumors; however, these did not remain significant after correction for FDR (supplementary table 5).

The median number of involved pathways was 4 with $90 \%$ of patients having at least two affected pathways 
Table 2 Summary of key involved pathways by disease status at time of sequencing

\begin{tabular}{|c|c|c|c|c|c|c|}
\hline & [ALL] & de novo & $R / R$ & $\mathrm{tFL}$ & $p$ value & $\mathrm{BH} p^{*}$ \\
\hline & 198 & 114 & 58 & 26 & & \\
\hline Tumor suppression & $123(62.1 \%)$ & $61(53.5 \%)$ & 44 (75.9\%) & $18(69.2 \%)$ & 0.007 & 0.079 \\
\hline Histone epigenetic & 107 (54.0\%) & 47 (41.2\%) & 37 (63.8\%) & $23(88.5 \%)$ & 0.008 & 0.079 \\
\hline BCR NFKB & $107(54.0 \%)$ & $60(52.6 \%)$ & $30(51.7 \%)$ & $17(65.4 \%)$ & 1.000 & 1.000 \\
\hline Transcription factors & $74(37.4 \%)$ & $44(38.6 \%)$ & $23(39.7 \%)$ & 7 (26.9\%) & 1.000 & 1.000 \\
\hline Cell death & $68(34.3 \%)$ & $27(23.7 \%)$ & 24 (41.4\%) & 17 (65.4\%) & 0.026 & 0.165 \\
\hline Immune evasion & $53(26.8 \%)$ & $30(26.3 \%)$ & $14(24.1 \%)$ & $9(34.6 \%)$ & 0.901 & 1.000 \\
\hline NOTCH MYC & $45(22.7 \%)$ & $22(19.3 \%)$ & $14(24.1 \%)$ & $9(34.6 \%)$ & 0.590 & 0.991 \\
\hline JAK STAT & $35(17.7 \%)$ & $22(19.3 \%)$ & $11(19.0 \%)$ & $2(7.7 \%)$ & 1.000 & 1.000 \\
\hline RAS MAPK & $35(17.7 \%)$ & $21(18.4 \%)$ & $8(13.8 \%)$ & $6(23.1 \%)$ & 0.582 & 0.991 \\
\hline Metabolism & $32(16.2 \%)$ & $18(15.8 \%)$ & 7 (12.1\%) & 7 (26.9\%) & 0.670 & 0.991 \\
\hline Cell cycle & $24(12.1 \%)$ & $15(13.2 \%)$ & $5(8.6 \%)$ & $4(15.4 \%)$ & 0.531 & 0.991 \\
\hline Translation & $22(11.1 \%)$ & $9(7.9 \%)$ & $9(15.5 \%)$ & $4(15.4 \%)$ & 0.200 & 0.635 \\
\hline SWI SNF epigenetic & $21(10.6 \%)$ & $12(10.5 \%)$ & $9(15.5 \%)$ & $0(0.0 \%)$ & 0.485 & 1.000 \\
\hline DNA damage & $21(10.6 \%)$ & $13(11.4 \%)$ & $6(10.3 \%)$ & $2(7.7 \%)$ & 1.000 & 0.991 \\
\hline RNA processing & $20(10.1 \%)$ & $14(12.3 \%)$ & $5(8.6 \%)$ & $1(3.8 \%)$ & 0.641 & 0.991 \\
\hline Epigenetic cofactors & $19(9.6 \%)$ & $8(7.0 \%)$ & $10(17.2 \%)$ & $1(3.8 \%)$ & 0.071 & 0.269 \\
\hline PI3K AKT TOR & $17(8.6 \%)$ & $10(8.8 \%)$ & $7(12.1 \%)$ & $0(0.0 \%)$ & 0.678 & 0.991 \\
\hline DNA epigenetic & $13(6.6 \%)$ & $11(9.6 \%)$ & $1(1.7 \%)$ & $1(3.8 \%)$ & 0.062 & 0.269 \\
\hline Adhesion cytoskeleton & $12(6.1 \%)$ & $9(7.9 \%)$ & $3(5.2 \%)$ & $0(0.0 \%)$ & 0.753 & 1.000 \\
\hline
\end{tabular}

Only alterations observed within at least ten patients are included

Differing values with unadjusted $p<0.05$ depicted in bold

$R / R$ relapsed refractory, $S N V$ short nucleotide variant, $t F L$ transformed follicular lymphoma

${ }^{*} p$ values reflect the comparison of $\mathrm{R} / \mathrm{R}$ to de novo disease (i.e. excludes $\mathrm{tFL}$ ).

(Table 2, Fig. 2). Overall, 90\% $(n=179)$ of the patients harbored a GA which could be explored for therapeutic intervention, with $54 \%(n=107)$ harboring more than one potential target. GAs involving a gene that is targeted by a drug approved by the FDA for another indication (level $2 B)$ were identified in $56 \%(n=110)$. These were comprised mainly of BCL2 inhibitors for BCL2-associated GAs, BTK inhibitors for MYD88mut, BRAF inhibitors for BRAFmut and immune check-point inhibitors in CD274 and PDCD1LG2 GAs (supplementary excel file 2). In $41 \%$ $(n=81)$ there was a GA targeted by a non-FDA-approved drug with compelling clinical evidence either in DLBCL (level $3 \mathrm{~A} ; 33 \%, n=66$; mostly histone deacetylase and EZH2 inhibitors in CREBBPmut, EP300mut, and EZH2$m u t$ ) or in another indication (level 3B; $8 \%, n=15$ ). Finally, in $85 \%$ there was at least one target for a drug in preclinical or early clinical development (level 4).

\section{Association of GAs with clinical presentations and pathologic subtypes}

There were 106 de novo DLBCL patients treated with an RCHOP-based chemotherapy (58\% RCOHP, 36\%
RCHOP-ICE, 7\% DA-EPOCH-R). Median age was 58 (range 21-84) with a $61 \%$ male predominance. The majority (75\%) had a stage III-IV disease, and 35\% had an intermediate-high to high IPI. Complete response was observed in $88 \%$ ( $\mathrm{PR}$ in $3 \%$ and $\mathrm{SD} / \mathrm{PD}$ in $9 \%$ ) of the patients and was not associated with stage, IPI, or other demographics (Table 3). Median follow-up was 66 months (95\% CI 57-73) with 18 deaths and 26 disease progressions documented during that time, representing a $5 y$ OS of $84 \%$ (95\%CI 77-92\%).

We investigated whether specific GAs were associated with patient outcomes, including response to chemotherapy or OS. We found that TP53 alterations predicted for lack of response to chemotherapy. Of the 12 patients with primary refractory disease, $8(67 \%)$ were TP53mut. Of the 19 patients harboring a TP53mut, 10 (53\%) did not achieve a complete response (CR) or relapsed within the first year, while one patient relapsed after 3 years. Six were without evidence of disease during a 2-5-year-follow-up, and two were still in remission at 12 and 22 months. TP53mut was also associated with shorter OS (5yOS $61 \%$ vs. $89 \%$ HR 5.8 95\%CI $2.1-16, p=0.001)$. 


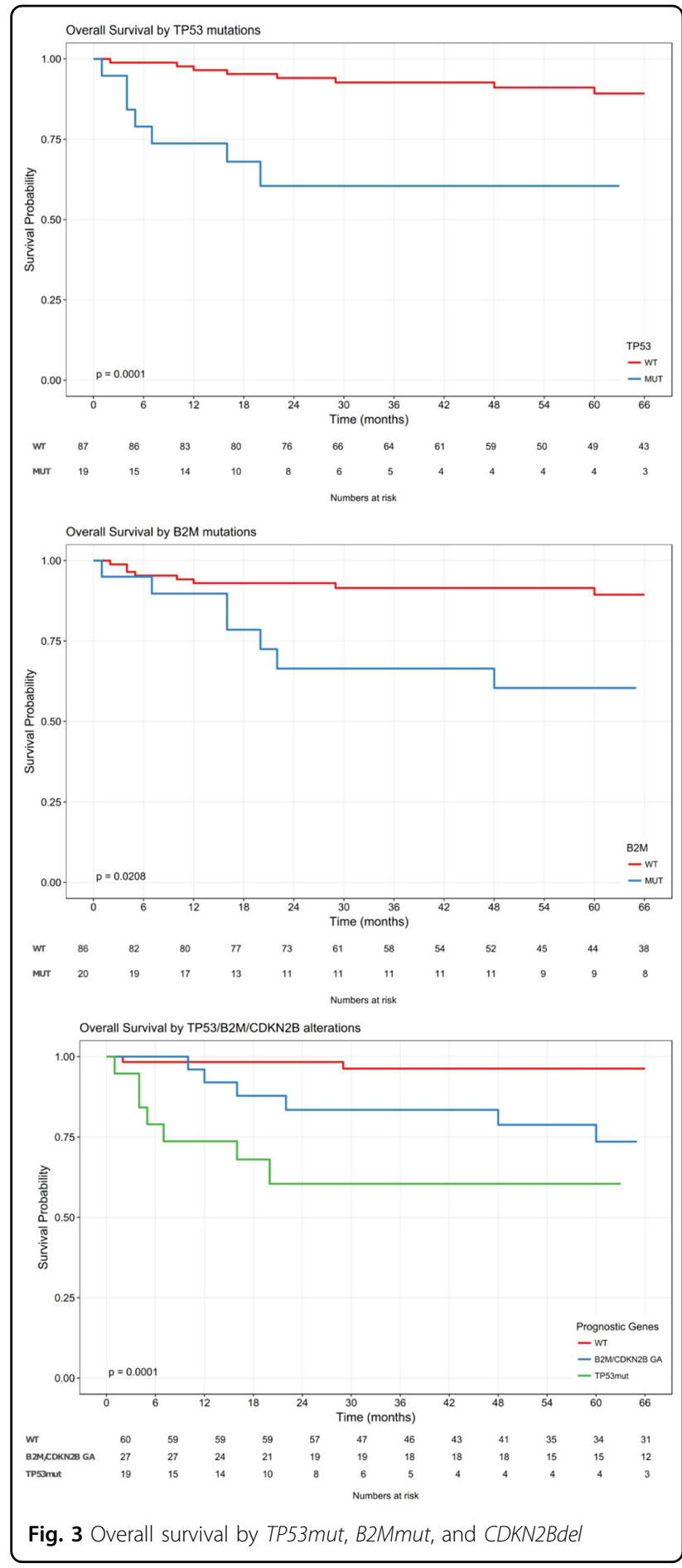

Finally, TP53mut were detected in $19 \mathrm{R} / \mathrm{R}$ patients, of whom $13(66 \%)$ were either refractory to their previous frontline therapy or had relapsed within less than a year.

Two other GAs were marginally associated with survival. B2Mmut (19\% of patients; OS HR 2.9, 95\%CI 1.1-7.6, $p=0.03)$, and CDKN2Adel when accompanied by CDKN2Bdel but not alone (14\% of patients, OS HR 2.5
Table 3 Baseline characteristic of de novo RCHOP/ RCHOP-like treated patients by CR attainment

\begin{tabular}{|c|c|c|c|c|}
\hline & [ALL] & CR & $\mathrm{PR} / \mathrm{SD} / \mathrm{PD}$ & $p$ value \\
\hline & $106^{*}$ & 93 & 12 & \\
\hline Age (min, max) & $58(21,84)$ & $58(21,83)$ & $51(31,84)$ & 0.774 \\
\hline Age $>65$ & $28(26.4 \%)$ & $25(26.9 \%)$ & $3(25.0 \%)$ & $>0.999$ \\
\hline $\operatorname{Sex}(M)$ & $65(61.3 \%)$ & $56(60.2 \%)$ & $8(66.7 \%)$ & 0.907 \\
\hline Disease status & & & & 0.320 \\
\hline de novo & $96(90.6 \%)$ & 85 (91.4\%) & $10(83.3 \%)$ & \\
\hline $\begin{array}{c}\text { tFL (first } \\
\text { presentation) }\end{array}$ & $10(9.4 \%)$ & $8(8.6 \%)$ & $2(16.7 \%)$ & \\
\hline Stage & & & & $>0.999$ \\
\hline$|/| \mid$ & 27 (25.5\%) & $24(25.8 \%)$ & $3(25.0 \%)$ & \\
\hline III/IV & 79 (74.5\%) & 69 (74.2\%) & $9(75.0 \%)$ & \\
\hline$|P| \geq 3$ & 37 (34.9\%) & 31 (33.3\%) & $6(50.0 \%)$ & 0.337 \\
\hline Cell of origin & & & & 0.815 \\
\hline GCB & $54(50.9 \%)$ & $48(51.6 \%)$ & $5(41.7 \%)$ & \\
\hline Non-GCB & 41 (38.7\%) & $35(37.6 \%)$ & $6(50.0 \%)$ & \\
\hline Unclassified & 11 (10.4\%) & $10(10.8 \%)$ & $1(8.3 \%)$ & \\
\hline Treatment & & & & $<0.001$ \\
\hline DAEPOCHR & $7(6.6 \%)$ & $3(3.2 \%)$ & $4(33.3 \%)$ & \\
\hline $\mathrm{RCHOP}$ & $61(57.5 \%)$ & 52 (55.9\%) & $8(66.7 \%)$ & \\
\hline RCHOP_ICE & 38 (35.8\%) & $38(40.9 \%)$ & $0(0.0 \%)$ & \\
\hline
\end{tabular}

*One patient died during frontline therapy and did not have a response designation.

$C R / P R$ complete/partial response, SD/PD stable/progressive disease

95\%CI 0.9-7.1, $p=0.08$ ). (Table 4, Fig. 3). In addition, abnormalities grouped under the protein translation machinery pathway (e.g. $M Y C, E I F 4 A 2$ ), present in $9 \%$ of patients, were associated with a shorter OS (HR 4.9 95\%CI 1.7-13.8, $p=0.003)$, as were abnormalities in tumor suppressor pathways (which includes TP53mut). Lastly, patients with no alteration in TP53, CDKN2B or B2M, had a remarkably long OS (5yOS $96 \%$ vs. $74 \%$ for B2Mmut/CDKN2Bdel $p=0.03$, and $60 \%$ for TP53mut $p$ $<0.0001$ ) (Fig. 3).

\section{Discussion}

Clinical trials that select patients for novel targeted therapies based on GAs require large-scale standardized sequencing endeavors, which can be facilitated by targeted DNA and RNA sequencing of FFPE specimens. This work describes the application of a CLIA-compliant integrated DNA and RNA targeted sequencing panel to a retrospective cohort of 198 FFPE DLBCL specimens for the identification of GAs with potential clinical significance. 
Table 4 GAs and pathways associated with response and/or survival

\begin{tabular}{|c|c|c|c|c|c|c|c|}
\hline Gene & $N$ & HR PFS $(95 \% \mathrm{Cl})$ & $p$ value & $\mathrm{BH} p$ & HR OS $(95 \% \mathrm{Cl})$ & $p$ value & $\mathrm{BH} p$ \\
\hline CDKN2A & 25 & $1.4(0.6,3.0)$ & 0.391 & 0.687 & $1.7(0.6,4.4)$ & 0.314 & 0.847 \\
\hline KMT2D & 25 & $1.8(0.9,3.8)$ & 0.103 & 0.428 & $1.2(0.4,3.3)$ & 0.754 & 0.896 \\
\hline$B C L 2$ & 21 & $1.9(0.9,4.0)$ & 0.114 & 0.428 & $0.9(0.3,3.1)$ & 0.853 & 0.896 \\
\hline$B 2 M$ & 20 & $1.4(0.6,3.1)$ & 0.483 & 0.725 & $2.9(1.1,7.6)$ & 0.027 & 0.204 \\
\hline$B C L 6$ & 19 & $0.4(0.1,1.4)$ & 0.161 & 0.482 & $0.9(0.3,3.1)$ & 0.866 & 0.896 \\
\hline TP53 & 19 & $4.5(2.1,9.5)$ & $<0.0001$ & 0.001 & $5.8(2.1,16.0)$ & 0.001 & 0.011 \\
\hline CREBBP & 18 & $1.5(0.7,3.5)$ & 0.319 & 0.687 & $1.9(0.7,5.4)$ & 0.225 & 0.844 \\
\hline MYD88 & 18 & $1.4(0.6,3.3)$ & 0.408 & 0.687 & $0.6(0.1,2.7)$ & 0.534 & 0.847 \\
\hline CDKN2B & 15 & $2.2(0.9,5.0)$ & 0.076 & 0.428 & $2.5(0.9,7.1)$ & 0.079 & 0.395 \\
\hline TNFAIP3 & 15 & $0.7(0.2,2.3)$ & 0.569 & 0.764 & $1.4(0.4,5.0)$ & 0.564 & 0.847 \\
\hline$E Z H 2$ & 14 & $0.7(0.2,2.4)$ & 0.611 & 0.764 & $0.4(0.1,3.2)$ & 0.411 & 0.847 \\
\hline REL & 12 & $0.5(0.1,2.3)$ & 0.412 & 0.687 & $0.9(0.2,3.9)$ & 0.896 & 0.896 \\
\hline PIM1 & 11 & $0.9(0.3,2.8)$ & 0.800 & 0.858 & $0.4(0.0,2.9)$ & 0.348 & 0.847 \\
\hline TNFRSF14 & 11 & $1.0(0.3,3.4)$ & 0.972 & 0.972 & $0.6(0.1,4.2)$ & 0.565 & 0.847 \\
\hline \multicolumn{8}{|l|}{ Pathway } \\
\hline Translation & 10 & $2.3(0.9,6.0)$ & 0.091 & 0.453 & $4.9(1.7,13.8)$ & 0.003 & 0.039 \\
\hline Tumor suppressor/p53 & 56 & $3.2(1.5,7.0)$ & 0.003 & 0.052 & $4.3(1.4,13.2)$ & 0.011 & 0.081 \\
\hline Immune evasion & 31 & $1.1(0.5,2.3)$ & 0.886 & 0.961 & $2.2(0.9,5.6)$ & 0.095 & 0.473 \\
\hline Epigenetic histone all & 49 & $1.9(0.9,3.8)$ & 0.083 & 0.453 & $1.9(0.7,4.9)$ & 0.184 & 0.689 \\
\hline
\end{tabular}

Differing values with unadjusted $p<0.05$ depicted in bold

$B H$ FDR-adjusted $p$ value (Benjamini-Hochberg), $R / R$ relapsed refractory, SNV short nucleotide variant, $t F L$ transformed follicular lymphoma

Prior studies defining the genomic landscape of DLBCL have produced highly variable results ${ }^{2}$. For example, reported frequencies of CREBBP/EP300 mutations in DLBCL have ranged widely from 5 to $44 \%^{3-7}$. We detected alterations in the histone acetyltransferases $C R E B B P / E P 300$ in $21 \%$ of clinical FFPE DLBCL specimens. This frequency corresponds to the approximate $20 \%$ response rates to HDAC inhibitors observed in unselected patients with DLBCL, providing a biologic rationale for a clinical trial using CREBBP/EP300 mutation as a genomic biomarker to select DLBCL patients for treatment with the HDAC inhibitors (NCT02282358) ${ }^{19}$. Likewise, prior studies reported EZH2 mutations frequencies as high as $24 \%{ }^{20}$, whereas we found $E Z H 2 m u t$ in $11 \%$ of our cohort, a difference that would have major implications for designing a trial with sequencing-based selection of patients for treatment with EZH2 inhibitors.

Comprehensive genomic profiling also detected uncommon GAs (occurring at $<3 \%$ frequency) with potential therapeutic relevance, which could help identify patients for genetically defined basket trials or select molecularly targeted therapies (supplementary excel file 2). For example, BRAF V600E and L597R/V mutations which may predict for response to BRAF or MEK inhibitors $^{21}$. Likewise, we identified mutations in ID3 (L70P, P98R, Q100P) and TCF3 (N551K), which had been previously described, predominantly in Burkitt lymphoma, to confer dependence on PI3K pathway signaling, and may indicate a potential therapeutic vulnerability ${ }^{22}$.

In our study, $90 \%$ of the patients had at least one potentially targetable GA which could guide selection for clinical trials. Nearly two-thirds of these patients harbored two or more such targets. When using genomic profiling to define genomic biomarkers, it will be important to determine how variant allele frequencies (VAFs) of actionable mutations impact responses to molecularly targeted therapies. For example, we found that gain-offunction mutations in MYD88 L273P (a.k.a. L265P) exhibited VAFs that ranged from 9 to $74 \%$ (supplementary table 3). Thus, if the MYD 88 mutations were targeted with a downstream IRAK1/4 inhibitor, the question arises as to whether tumors showing subclonal GAs with low VAFs will respond similarly to those with a high VAF dominant clone $^{23}$. Although the impact of clonal and subclonal GAs on response to targeted therapies will become clearer as empiric observations are gathered, 
consensus regarding this question will need to be established soon, in order to design clinical trials that prospectively select patients based on genomic biomarkers ${ }^{16}$.

Consistent with previous reports, using non-clinical genome sequencing assays in the research setting, we confirmed that KMT2Dmut to be the most common SNV, present in $26 \%$ of de novo cases $^{3-5,7,24,25}$. Similarly, TP53mut was noted in $16 \%$ of de novo disease and was enriched among $\mathrm{R} / \mathrm{R}$ patients $(33 \%)^{3-5,24,25}$. Further, in agreement with previous literature, certain GAs were highly associated with cell of origin designation, highlighting the need to account for molecular case-mix when comparing results from different genomic profiling studies. Compared to non-GCB, GCB samples were enriched for BCL2trans, CREBBPmut, KMT2Dmut, TNFRSF14mut, and EZH2mut, in keeping with rates described previously in the literature ${ }^{6,720,24}$. Likewise, non-GCB samples were enriched for CD79Bmut, BCL6trans, MYD88mut, ETV6mut, and PRDM1mut also at similar rates as previously described ${ }^{6,7,20,24}$.

Despite an accumulating body of research into the genomic landscape of DLBCL, very few GAs have been found to be associated with treatment refractoriness or disease relapse. Our study confirms prior associations between TP53mut and survival ${ }^{26,27}$. Though marginally significant, $C D K N 2 A / B d e l$ and $B 2 M m u t$ were also found to be associated with shorter $\mathrm{OS}^{28-30}$.

As larger sequencing cohorts are assembled, future studies will continue to refine the association between GAs and treatment outcomes. While many large centers use their own home-grown sequencing assays to select patients for targeted therapy, the use of a commercially available clinical assay can facilitate the application of precision medicine at local hospitals and doctor offices, in addition to facilitating the conduct of clinical trials across several institutions. Furthermore, the use of a standardized commercial assay may allow comparing the results of different clinical trials that select patients based on specific genetic alterations. Typically, the cost of a commercial assay is higher than institutional platforms. However, only large institutions are able to develop their own sequencing assays, leaving smaller centers to depend on third-party commercial vendors.

\section{Acknowledgements}

We thank the patients at Memorial Sloan Kettering Cancer Center who generously donated their biopsy specimens for research. We thank members of the Lymphoma Service at Memorial Sloan Kettering Cancer Center for helpful discussions and feedback. A.M.I. is supported by the NIH/NCI (K08 CA201483), Leukemia \& Lymphoma Society (Special Fellow Award 3356-16), Burroughs Welcome Fund (Career Award for Medical Scientists 1015584), Susan and Peter Solomon Divisional Genomics Program, and Steven A. Greenberg Fund. The work was supported in part by the John and Barbara Vogelstein Foundation, the Memorial Sloan Kettering Cancer Center Support Grant NIH P30 CA008748, and the MSK SPORE in lymphoma (P50 CA192937). We thank Foundation Medicine, Inc., for performing the targeted sequencing assays as a collaborative research effort.

\section{Author details}

'Lymphoma Service, Department of Medicine, Memorial Sloan Kettering Cancer Center, New York, NY, USA. ²Department of Medicine, Weill Cornell Medical College, New York, NY, USA. ${ }^{3}$ Human Oncology and Pathogenesis Program, Memorial Sloan Kettering Cancer Center, New York, NY, USA. ${ }^{4}$ Center for Hematologic Malignancies, Memorial Sloan Kettering Cancer Center, New York, NY, USA. ${ }^{5}$ Department of Epidemiology and Biostatistics, Memorial Sloan Kettering Cancer Center, New York, NY, USA. ${ }^{6}$ Foundation Medicine Inc., Cambridge, MA, USA. ${ }^{7}$ Division of Hematologic Oncology, Memorial Sloan Kettering Cancer Center, New York, NY, USA. ${ }^{8}$ Department of Pathology, Memorial Sloan Kettering Cancer Center, New York, NY, USA. 'Leukemia Service, Department of Medicine, Memorial Sloan Kettering Cancer Center, New York, NY, USA

\section{Authors' contributions}

A.M.I., E.J., and A.Y. conceived the project, analyzed the data, and wrote the manuscript. A.M.I., R.L.L., A.D.Z., A.D., M.L.P., M.R.v.d.B., and A.Y. helped design the content for the sequencing assay. J.H., M.K.N., S.E.T., T.M., and V.A.M. constructed and validated the sequencing assay, processed the samples, and performed the sequencing. P.H., E.J., and V.E.S. performed the statistical analysis. E.P. and M.M. assisted with bioinformatics analyses. A.R.C., A.D., C.L.B., C.H.M., C.P., D.J.S., M.M., A.N., S.H., J.F.G., A.M., P.H., A.K., K.M.K., and J.D.P. assisted with logistics of sample identification/collection, assessment of clinical data, and pathologic review.

\section{Conflict of interest}

A.M.I., R.L.L., A.D.Z., A.D., M.L.P., M.R.v.d.B., and A.Y. have served as consultants for Foundation Medicine, Inc. J.H., M.K.N., S.E.T., and V.A.M. are employees of Foundation Medicine, Inc. V.A.M. has ownership interest (including patents) in Foundation Medicine, Inc. The remaining authors declare that they have no conflict of interest.

\section{Publisher's note}

Springer Nature remains neutral with regard to jurisdictional claims in published maps and institutional affiliations.

Supplementary Information accompanies this paper at (https://doi.org/ 10.1038/s41408-018-0089-0).

Received: 21 January 2018 Revised: 12 March 2018 Accepted: 29 March 2018

Published online: 12 June 2018

\section{References}

1. Garraway, L. A. Genomics-driven oncology: framework for an emerging paradigm. J. Clin. Oncol. 31, 1806-1814 (2013).

2. Roschewski, M., Staudt, L. M. \& Wilson, W. H. Diffuse large B-cell lymphomatreatment approaches in the molecular era. Nat. Rev. Clin. Oncol. 11, 12-23 (2014).

3. Lohr, J. G. et al. Discovery and prioritization of somatic mutations in diffuse large B-cell lymphoma (DLBCL) by whole-exome sequencing. Proc. Natl. Acad. SCi. USA 109, 3879-3884 (2012).

4. Morin, R. D. et al. Mutational and structural analysis of diffuse large B-cell lymphoma using whole-genome sequencing. Blood 122, 1256-1265 (2013).

5. Pasqualucci, L. et al. Analysis of the coding genome of diffuse large B-cell lymphoma. Nat. Genet. 43, 830-837 (2011).

6. Zhang, J. et al. Genetic heterogeneity of diffuse large B-cell lymphoma. Proc. Natl. Acad. Sci. USA 110, 1398-1403 (2013).

7. Reddy, A. et al. Genetic and functional drivers of diffuse large B cell lymphoma. Cell 171, 481-494.e15 (2017).

8. Dubois, S.., \& Jardin, F.. The role of next-generation sequencing in understanding the genomic basis of diffuse large B cell lymphoma and advancing targeted therapies. Expert Rev. Hematol. 4086, 1-15 (2016).

9. Zehir, A. et al. Mutational landscape of metastatic cancer revealed from prospective clinical sequencing of 10,000 patients. Nat. Med. 23, 703-713 (2017). 
10. He, J. et al. Integrated genomic DNA/RNA profiling of hematologic malignancies in the clinical setting. Blood 127 (24), 3004-14 (2016).

11. Frampton, G. M. et al. Development and validation of a clinical cancer genomic profiling test based on massively parallel DNA sequencing. Nat. Biotechnol. 31, 1023-1031 (2013).

12. Younes, A. et al. The landscape of new drugs in lymphoma. Nat. Rev. Clin. Oncol. 14, 335-346 (2017).

13. Intlekofer, A. M. \& Younes, A. Precision therapy for lymphoma? Current state and future directions. Nat. Rev. Clin. Oncol. 11, 585-596 (2014).

14. Chakravarty D., Gao J., Phillips S. \& Kundra R. OncoKB: a precision oncology knowledge base. Precis Oncol. 2017; e-pub ahead of print.

15. Genomics - Table of Pharmacogenomic Biomarkers in Drug Labeling. https:// www.fda.gov/drugs/scienceresearch/researchareas/pharmacogenetics/ ucm083378.htm. Accessed September 1, 2017.

16. Joffe, E., lasonos, A. \& Younes, A. Clinical trials in the genomic era. J. Clin. Oncol. 35, 1011-1017 (2017).

17. GeneCards_-Human Genes Database. http://www.genecards.org/. Accessed September 23, 2017.

18. Guirguis, H. R. et al. Survival of patients with transformed lymphoma in the rituximab era. Ann. Hematol. 93, 1007-1014 (2014).

19. Lane, A. A. \& Chabner, B. A. Histone deacetylase inhibitors in cancer therapy. J. Clin. Oncol. 27, 5459-5468 (2009).

20. Morin, R. D. et al. Frequent mutation of histone-modifying genes in nonHodgkin lymphoma. Nature 476, 298-303 (2011).

21. Holderfield, M., Deuker, M. M., McCormick, F. \& McMahon, M. Targeting RAF kinases for cancer therapy: BRAF-mutated melanoma and beyond. Nat. Rev Cancer 14, 455-467 (2014)
22. Schmitz, R. et al. Burkitt lymphoma pathogenesis and therapeutic targets from structural and functional genomics. Nature 490, 116-120 (2012).

23. Ngo, V. N. et al. Oncogenically active MYD88 mutations in human lymphoma Nature 470, 115-119 (2011).

24. Dubois, S. et al. Next-generation sequencing in diffuse large B-cell lymphoma highlights molecular divergence and therapeutic opportunities: a LYSA study. Clin. Cancer Res. 22, 2919-2928 (2016).

25. Melchardt, T. et al. Clonal evolution in relapsed and refractory diffuse large Bcell lymphoma is characterized by high dynamics of subclones. Oncotarget $\mathbf{5}$, 51494-51502 (2016).

26. Xu-Monette, Z. Y. et al. Mutational profile and prognostic significance of TP53 in diffuse large B-cell lymphoma patients treated with rituximab-CHOP: a report from an International DLBCL Rituximab-CHOP Consortium Program study. Blood 120, 3986-3997 (2012)

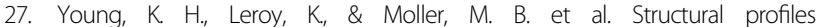
of TP53 gene mutations predict clinical outcome in diffuse large B-cel lymphoma: an international collaborative study. Blood 112, 3088-3098 (2008).

28. Jardin, F. et al. Diffuse large B-cell lymphomas with CDKN2A deletion have a distinct gene expression signature and a poor prognosis under R-CHOP treatment: a GELA study. Blood 116, 1092-1104 (2010).

29. Challa-Malladi, M. et al. Combined genetic inactivation of $\beta 2$-microglobulin and CD58 reveals frequent escape from immune recognition in diffuse large $B$ cell lymphoma. Cancer Cell 20, 728-740 (2011).

30. Barrans, S. et al. Rearrangement of MYC is associated with poor prognosis in patients with diffuse large B-cell lymphoma treated in the era of rituximab. J. Clin. Oncol. 28, 3360-3365 (2010). 